\title{
EVALUACIÓN DE LAS FUNCIONES EJECUTIVAS EN ADULTOS CON DISCAPACIDAD INTELECTUAL: IMPLICACIONES PARA EL APRENDIZAJE A LO LARGO DE LA VIDA
}

\author{
Marta Bestué Laguna \\ Facultad Ciencias Humanas y de la Educación. Universidad de Zaragoza mbestue@unizar.es \\ Elena Escolano-Pérez \\ Facultad de Educación. Universidad de Zaragoza. eescola@unizar.es \\ María Antonia Acero Ferrero \\ Facultad de Educación. Universidad de Zaragoza. macero@unizar.es
}

Recepción Artículo: 27 octubre 2021 Admisión Evaluación: 27 octubre 2021 Informe Evaluador 1: 28 octubre 2021 Informe Evaluador 2: 29 octubre 2021 Aprobación Publicación: 30 octubre 2021

\section{RESUMEN}

Las funciones ejecutivas son procesos cognitivos de alto nivel que permiten adaptarnos a las situaciones complejas y novedosas. Son numerosos los trastornos y discapacidades en las que aparecen afectadas, limitando la calidad de vida de estas personas. El objetivo de este estudio fue evaluar las funciones ejecutivas de adultos que presentaban distintas discapacidades y trastornos, para a partir de esta evaluación, diseñar en el ámbito de la educación no formal un programa de intervención destinado a su mejora. Participaron 11 adultos de edades comprendidas entre los 22 y 54 años (media $=34,5$ ) y que presentaban las siguientes discapacidades/trastornos: discapacidad intelectual con un grado de dependencia desde el $34 \%$ hasta un $65 \%$. Todos ellos eran miembros de una misma asociación. En cada uno de los participantes se evaluaron individualmente los componentes ejecutivos de memoria de trabajo verbal y no verbal, resistencia a la interferencia, inhibición conductual y flexibilidad cognitiva a través de las tareas de dígitos inversos, cubos de Corsi, Caras, Estatua y clasificación de cartas, respectivamente. Los resultados muestran que la inhibición es el componente ejecutivo más afectado en la muestra, seguido de la memoria de trabajo no verbal, la memoria de trabajo verbal y la flexibilidad. Estos resultados ofrecen una evaluación inicial del funcionamiento ejecutivo de los participantes, resultando útiles para el diseño de un programa de intervención en el ámbito de la educación no formal que responda a sus necesidades. La finalidad última de todo ello es contribuir al desarrollo de la autonomía de los participantes para una mejora de su calidad de vida.

Palabras clave: funciones ejecutivas; discapacidad; intervención; educación no formal

\section{ABSTRACT}

Assessment of executive functions in adults with intellectual disabilities: implications for lifelong learning. Executive functions are high-level cognitive processes that allow us to adapt to complex and 


\section{EVALUACIÓN DE LAS FUNCIONES EJECUTIVAS EN ADULTOS CON DISCAPACIDAD INTELECTUAL: IMPLICACIONES PARA EL APRENDIZAJE A LO LARGO DE LA VIDA}

novel situations. There are numerous disorders and disabilities in which they are affected, limiting the quality of life of these people. The objective of this study was to evaluate the executive functions of adults with different disabilities and disorders, and based on this evaluation, to design an intervention program aimed at their improvement in the field of non-formal education. Eleven adults between the ages of 22 and 54 (mean

$=34.5)$ participated and presented the following disabilities / disorders: intellectual disability with a degree of dependency ranging from $34 \%$ to $65 \%$. They were all members of the same association. In each of the participants, the executive components of verbal and non-verbal working memory, resistance to interference, behavioral inhibition and cognitive flexibility were individually evaluated through the tasks of inverse digits, Corsi's cubes, Faces, Statue and classification of letters, respectively. The results show that inhibition is the executive component most affected in the sample, followed by non- verbal working memory, verbal working memory, and flexibility. These results offer an initial evaluation of the executive functioning of the participants, being useful for the design of an intervention program in the field of non-formal education that responds to their needs. The ultimate purpose of all this is to contribute to the development of the autonomy of the participants to improve their quality of life.

Keywords: executive functions; disability; intervention; non formal education

\section{INTRODUCCIÓN}

Las funciones ejecutivas tienen una gran relación con muchos de los procesos implicados en el aprendizaje. Gracias a técnicas de neuroimagen hemos podido identificar cómo se activan las diferentes partes del cerebro correspondientes a los distintos elementos que componen las funciones ejecutivas. Por otro lado, existen muchos estudios (Cole et al., 2013 y Miyake y Friedman, 2012) que hacen hincapié en un modelo holístico en el que todos los componentes de las funciones ejecutivas quedan relacionados al trabajarse de forma independiente.

En este estudio se pretende analizar la situación de partida para plantear intervenciones para la mejora del funcionamiento ejecutivo de los participantes. Cuando se diseñan estas intervenciones se suelen desarrollar tareas que entrenan cada uno de los componentes, sin embargo, teniendo en cuenta el modelo integral, conocemos que se dan conexiones neuronales entre las diferentes áreas que comprometen los diferentes componentes (Derrfuss, Brass, Neumann y von Cramon, 2005).

Las funciones ejecutivas para autores como García-Molina, Enseñat-Cantallops, Tirapu- Ustárroz y RoigRovira (2009) son procesos cognitivos que permiten el control y regulación de comportamientos enfocados a una meta. Otros autores como Escolano- Pérez y Bravo (2017), Carlson, Zelazo y Faja, (2013), Diamond (2013) y Miyake y Friedman (2012) como un conjunto de competencias de carácter cognitivo y emocional que se encuentran vinculadas al desarrollo madurativo del cerebro y donde intervienen capacidades de autorregulación, planificación, flexibilidad y memoria de trabajo con el fin de adaptar las conductas a las situaciones en las que tengamos que dar una respuesta para alcanzar el objetivo.

Zelazo y Carlson (2012) reflejan en su estudio la diferencia entre las funciones ejecutivas hot y las cool. Siendo las hot todos aquellos elementos que están relacionados con las competencias emocionales y la cool las relacionadas con aspectos cognitivos.

Para este estudio, las funciones ejecutivas se van a abordar desde un modelo cognitivo, teniendo en cuenta que todos los componentes de las mismas quedan interrelacionados en el momento en el que se realizan intervenciones sobre uno de ellos. Es esperable que la mejora de uno de los componentes ejecutivos pueda conducir a mejoras en dominios diferentes debido al principio de transferencia (Doebel, 2020; Everts et al., 2019)

En cuanto al desarrollo madurativo de las funciones ejecutivas también existen numerosos estudios que determinan que en edades tempranas se da un desarrollo exponencial y que en torno a la juventud adulta es cuando se llega al máximo desarrollo de las mismas siguiendo un patrón de desarrollo como de "U" invertida siendo a partir de los 50060 años cuando comienza el declive (Flores-Lázaro, Castillo-Preciado y Jiménez-Miramonte, 2014 y Escolano-Pérez y Bravo-Álvarez, 2017). Sin embargo, teniendo en cuenta la plasticidad cerebral y que las funciones ejecutivas pueden ser entrenadas y mejoradas, su desarrollo puede optimizarse tanto en niños como 
en adultos a través de actividades concretas que requieran de la práctica de una función ejecutiva o de varias de ellas.

El desarrollo del funcionamiento ejecutivo tiene beneficios para toda la población, pero especialmente para aquellos que presentan dificultades en algunas de estas funciones, como por ejemplo personas con necesidades específicas de apoyo educativo derivadas de un Trastorno por Déficit de Atención, Trastorno de Espectro Autista, Discapacidad Intelectual, Trastorno Grave de Conducta o Trastorno de la Conducta Alimentaria entre otros.

En este estudio la discapacidad intelectual es una de las características de todos los participantes en el estudio. El diagnóstico temprano de discapacidad intelectual es importante de cara a una intervención precoz. En el caso de la población de este estudio se trata de personas adultas, las cuales han permanecido durante muchos años sin recibir una estimulación neurocognitiva que les permita desarrollar las funciones ejecutivas.

Rowe, Lavender y Turk en 2006 ya planteaban la necesidad de estimular las funciones ejecutivas de adultos con Síndrome de Down para la mejora de sus habilidades para la vida. Siguiendo esta investigación, en 2010, Danielsson, Henry, Rönnberg y Nilsson realizaron un estudio Iongitudinal de 5 años de entrenamiento de funciones ejecutivas con adultos con discapacidad intelectual obteniendo resultados positivos en las diferentes tareas planteadas.

La mejora del funcionamiento ejecutivo no solo tiene un impacto directo sobre el propio individuo en cuanto a mejora de la autoestima, mejora de condiciones laborales, mejora del nivel socioeconómico, desarrollo de competencias que conllevan una consecución de metas de manera eficaz, además existe una mejora del nivel social y económico del país donde se atienda a adultos con discapacidad intelectual proporcionándoles instrumentos para la reinserción laboral en determinados puestos de trabajo.

Estas mejoras sobre el funcionamiento ejecutivo repercuten directamente en el desarrollo de independencia de los adultos con discapacidad intelectual, por lo que la visibilización de este tipo de intervenciones debe ser cada vez más frecuente y podría ser uno de los pilares centrales en políticas de inclusión.

\section{OBJETIVOS DE LA INVESTIGACIÓN}

El objetivo de este estudio es determinar las dificultades del funcionamiento ejecutivo de los adultos con discapacidad intelectual para planificar una intervención basada en estas necesidades reales.

\section{MUESTRA Y/O PARTICIPANTES}

La muestra de este estudio la componen 10 adultos españoles con discapacidad intelectual de edades comprendidas entre los 22 y 54 años (media $=34,5$ ). No se realizan diferencias en cuanto al género ya que no se pueden extraer resultados generalizables teniendo en cuenta que 3 eran mujeres y 7 hombres. Todos los participantes forman parte de un programa de impulso para el empleo de estas personas con discapacidad.

\section{METODOLOGÍA Y/O INSTRUMENTOS UTILIZADOS}

Las tareas utilizadas para realizar una evaluación de las funciones ejecutivas (FE) según el modelo de Miyake y Friedman (2012) son las siguientes:

Memoria de trabajo: Se refiere a la capacidad de almacenar determinados elementos y ser capaz de mantenerlos en la memoria para utilizarlos de forma adecuada. Para ello se administró las subpruebas del WAIS IV (Weschler, 2012): dígitos directos, dígitos inversos y letras y números. Estas tareas evalúan la memoria de trabajo verbal o semántica. Para evaluar la memoria de trabajo visoespacial se realizó el test de cubos de Corsi (Corsi, 1973) partiendo de la numeración de los dígitos directos e inversos de las subpruebas del WAIS.

Inhibición: Estas tareas tienen como objetivo evaluar la capacidad de desestimar distractores para responder de forma adecuada. Las tareas seleccionadas para evaluar la inhibición conductual eran las del tipo Go No-Go (Börger y van der Meere, 2000). En esta tarea los participantes deben ser capaces de resistir la interferencia 0 un estímulo distractor. En este sentido se utilizó además la tarea de la estatua, subprueba del NEPSY-II (Korkman, Kirk y Kemp, 2014). Por otro lado, para evaluar los procesos atencionales relacionados con la inhibición cogni- 
tiva se realizó el test de percepción de diferencias (CARAS) de Thurstone y Yela (2012) el cual consta de 60 dibujos cada uno de ellos está formado por tres dibujos esquemáticos de caras con la boca, cejas y pelo representados con trazos elementales. La tarea consiste en desetimar la cara que es diferente.

Flexibilidad: Es la capacidad de modificar o flexibilizar una respuesta en función de la variación de la consigna aportada, por ejemplo, cambiar de forma rápida y eficaz el centro de atención, amoldándonos a las exigencias cambiantes de la tarea. Para evaluar este componente ejecutivo la tarea que se realizó fue el DCCS (Zelazo, 2006)

\section{RESULTADOS ALCANZADOS}

Para analizar los datos recogidos y poder establecer comparaciones entre las tareas realizadas se convirtieron las puntuaciones obtenidas a puntuaciones típicas, siendo 0 la más baja y 10 la más alta en todas las tareas.

A continuación, en la Tabla 1, se presentan los resultados del análisis descriptivo en función de las tareas realizadas.

Tabla 1. Estadísticos descriptivos.

\begin{tabular}{lccccc} 
& N & Mínimo & Máximo & Media & $\begin{array}{c}\text { Desv. } \\
\text { típ. }\end{array}$ \\
\hline Edad & 10 & 22 & 54 & 34,50 & 10,824 \\
\hline Dígitos directo & 10 & 1,88 & 6,25 & 4,0625 & 1,2926 \\
\hline Dígitos indirecto & 10 &, 00 & 5,63 & 3,4375 & 1,5095 \\
\hline $\begin{array}{l}\text { Letras y } \\
\text { números }\end{array}$ & 10 &, 33 & 6,33 & 3,6663 & 2,0240 \\
\hline $\begin{array}{l}\text { Go-NoGo } \\
\text { aciertos }\end{array}$ & 10 & 8,00 & 10,00 & 9,2133 &, 61728 \\
\hline Caras aciertos & 10 &, 17 & 3,83 & 1,9833 & 1,0814 \\
\hline Corsi directo & 10 & 1,88 & 4,38 & 3,0000 &, 87401 \\
\hline Corsi inverso & 10 &, 00 & 5,00 & 2,5625 & 1,6783 \\
\hline Estatua & 10 & 7,67 & 10,00 & 9,2667 &, 71665 \\
\hline DCCS & 10 & 2,00 & 7,00 & 5,1000 & 1,2866 \\
\hline
\end{tabular}

Se puede observar la puntuación media de cada una de las tareas, así como la puntuación máxima y la mínima y la desviación típica. 
Para facilitar la interpretación de los resultados anteriores en la Figura 1 se muestra un diagrama de caja 0 de bigotes donde aparecen representadas todas las puntuaciones de las diferentes tareas.

Figura 1. Diagrama de bigotes teniendo en cuenta las tareas de evaluación de las FE.

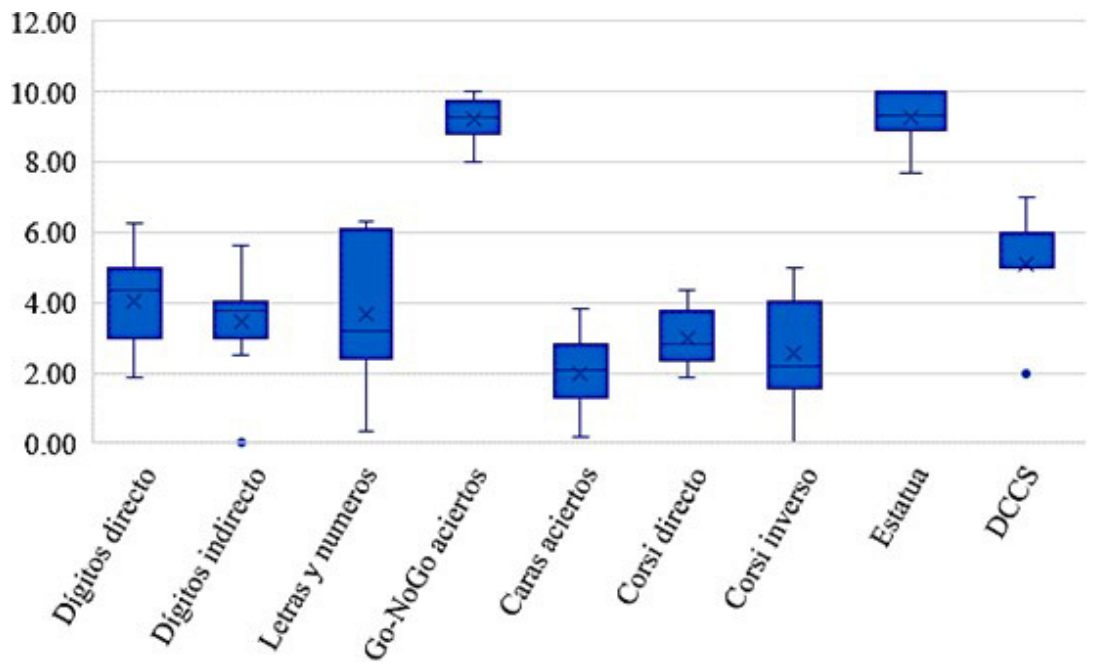

\section{DISCUSIÓN}

En la Figura 1 se puede observar cómo en la tarea de Go No-Go y en la de la estatua, ambas correspondientes a la inhibición conductual son las tareas mejor realizadas por los participantes, existiendo una clara diferencia con la tarea de atención del test de percepción de diferencias (Caras). Las puntuaciones medias más bajas, después del test de caras, son las correspondientes al componente ejecutivo de memoria de trabajo: Dígitos, letras y números y Corsi. En cuanto a la flexibilidad existen pocas diferencias entre las puntuaciones de los participantes y además se encuentra en la media de las puntuaciones del resto de tareas.

En otros estudios, como el de Gomes et al. (2021), encontraron diferencias en cuanto al rendimiento ejecutivo en función de la edad. Los bloques de edad eran de 18 a 30 años, de 31 a 45, de 46 a 59 y de 60 en adelante. En este caso contábamos con edades muy dispares con las que resulta difícil realizar generalizaciones o comparaciones con los resultados de otros estudios. Otro de los componentes que destacaba este estudio era la diferencia que se encontraba teniendo en cuenta el nivel de educación de los participantes. En nuestro estudio nos encontramos con adultos con discapacidad intelectual que abandonaron sus estudios de forma temprana, lo que plantea como necesario realizar una atención temprana y especialmente con personas con discapacidad intelectual ampliando su edad de escolaridad atendiendo a sus necesidades.

Estos resultados muestran la necesidad de realizar una intervención sobre el funcionamiento ejecutivo, haciendo hincapié en aquellas tareas en las que han obtenido peores resultados y utilizando sus puntos fuertes, como la inhibición conductual, para establecer el plan de intervención.

\section{CONCLUSIONES}

El aprendizaje a lo largo de la vida es una necesidad que debe estar presente en los diseños de intervención neuroeducativa de hoy en día. El entrenamiento de las funciones ejecutivas es una inversión a largo plazo que mejora las respuestas conductuales y emocionales adaptadas a las demandas que se nos exigen. En este caso 


\section{EVALUACIÓN DE LAS FUNCIONES EJECUTIVAS EN ADULTOS CON DISCAPACIDAD INTELECTUAL: IMPLICACIONES PARA EL APRENDIZAJE A LO LARGO DE LA VIDA}

estas habilidades les pueden resultar de gran utilidad en el camino hacia la inclusión y el desarrollo de su autonomía. La propuesta de intervención para la mejora de las FE debe darse desde los primeros años de vida hasta la adultez, siendo el componente de la plasticidad esencial en el desarrollo de las mismas.

Pero ¿qué implicación tiene una mejora de las funciones ejecutivas a lo largo de la vida? Si desglosamos cada uno de los componentes evaluados en este estudio comprenderemos la utilidad que tienen en las exigencias que subyacen en el día a día.

Para Diamond y Ling (2020) el control inhibitorio está presente en la vida cotidiana ya que tenemos que estar continuamente inhibiendo conductas o pensamientos que hagan que no seamos tan impulsivos y no tengamos que arrepentirnos después. La inhibición también está relacionada con la comprensión lectora, al necesitar inhibir la información innecesaria que afecte de forma negativa a nuestra comprensión. La memoria de trabajo se hace imprescindible ya que la necesitamos para almacenar determinados conceptos, consignas, números, etc. para ser más efectivos y eficaces en las tareas diarias. La flexibilidad también es importante en situaciones en las que debemos adaptarnos a las exigencias del medio, además juega un papel fundamental en la resolución de problemas siendo creativos y flexibles.

Este desarrollo de las FE les permitirá mejorar habilidades para la vida, especialmente en los casos en los que tienen un grado de dependencia asociado a la discapacidad intelectual. Con el desarrollo de las FE pueden llegar a sentirse capaces de realizar tareas por sí mismos de forma autónoma partiendo de estrategias aprendidas.

\section{REFERENCIAS BIBLIOGRÁFICAS}

Börger, N., y van der Meere, J. (2000). Motor control and state regulation in children with ADHD: a cardiac response study. Biological Psychology, 51(2-3), 247-267. doi:10.1016/s0301-0511(99)00040-x

Carlson, S.M., Zelazo, P.D., y Faja, S. (2013). Executive Function. Oxford Handbook of Developmental Psychology, Vol. 1 (pp. 706-742). doi:10.1093/0xfordhb/9780199958450.013.0025

Cole, M. W., Reynolds, J. R., Power, J. D., Repovs, G., Anticevic, A. y Braver, T. S. (2013). Multi-task connectivity reveals flexible hubs for adaptive task control. Nature Neuroscience, 16(9), 1348-1355. https://doi.org/10.1038/nn.3470

Corsi, P. M. (1973). Human memory and the medial temporal region of the brain.

Dissertation Abstracts International, 34(2-B), 891.

Danielsson, H., Henry, L., Rönnberg, J., \& Nilsson, L. G. (2010). Executive functions in individuals with intellectual disability. Research in Developmental Disabilities, 31(6), 1299-1304.

https://doi.org/10.1016/j.ridd.2010.07.012

Derffuss, J., Brass, M., Neumann, J. y von Cramon, D.Y. (2005), Involvement of the inferior frontal junction in cognitive control: Meta-analyses of switching and Stroop studies. Hum. Brain Mapp., 25: 22-34. https://doi.org/10.1002/hbm.20127

Diamond, A. (2013). Executive Functions. Annual Review of Psychology, 64, 135-168. Diamond, A. y Ling, S. (2020). Review of the evidence on, and Fundamental Questions

About efforts to improve Executive Functions including working memory. 145-

389. En Novick, J.; Bunting, M.; Dougherty, M. \&amp; Engle, R. (eds) Cognitive and Working memory Training: perspectives from psychology, neuroscience and human development. Oxford University Press.

Doebel, S. (2020). Rethinking Executive Function and Its Development. Perspectives on Psychological Science, 15(4), 942-956. https://doi.org/10.1177/1745691620904771

Escolano-Pérez, E. y Bravo, M. A. (2017). Procesos cognitivos y afectivos implicados en la resolución de problemas: desarrollo e intervención. Miscelánea Comillas, 75(146), 41-69.

Everts, R., Schöne, C. G., Mürner-Lavanchy, I., Lamp y Steinlin, M. (2019).

Development of executive functions from childhood to adolescence in very preterm-born individuals - A 
Iongitudinal study. Early Human Development, 129, 45-51.

https://doi.org/10.1016/i.earlhumdev.2018.12.012

Flores-Lázaro, J. C., Castillo-Preciado, R. E. y Jiménez-Miramonte, N. A. (2014).

Desarrollo de funciones ejecutivas, de la niñez a la juventud. Anales de psicología, 30(2), 463-473.

García-Molina, A., Enseñat-Cantallops, A., Tirapu-Ustárroz, J. y Roig-Rovira, T. (2009). Maduración de la corteza prefrontal y desarrollo de las funciones ejecutivas durante los primeros cinco años de vida. Revista de Neurología, 48(8), 435-440.

Gomes de Santana, Y. E., Bessa da Conceição, J. R., Cerqueira Caribé, G. L., Ramos, T., Costa, R., Atalaia-Silva, K. C., y Abreu, N. (2021). Normas do Cubos de Corsi para população adulta. Neuropsicologia Latinoamericana, 13(2), 1-10.

Korkman, M., Kirk, U. y Kemp. S. (2014). NEPSY-II: Batería Neuropsicológica Infantil. Segunda Edición. Madrid: Pearson

Miyake, A., y Friedman, N.P. (2012). The Nature and Organization of Individual Differences in Executive Functions, Four General Conclusions. Current Directions in Psychological Science, 21(1): 8-14.

Rowe, J., Lavender, A., y Turk, V. (2006). Cognitive executive function in Down's syndrome. British Journal of Clinical Psychology, 45(1), 5-17

Thurstone, L. L., y Yela, M. (2012). CARAS. Test de percepción de diferencias. Madrid: TEA ediciones.

Wechsler, D. (2012). WAIS-IV. Escala de inteligencia de Wechsler para adultos-IV. Manual de aplicación y corrección. Madrid: NCS Pearson, Inc. Edición original, 2008.

Zelazo, P. D. (2006). The Dimensional Change Card Sort (DCCS): A method of assessing executive function in children. Nature protocols, 1(1), 297-301.

Zelazo, P. D. y Carlson, S. M. (2012). Hot and Cool Executive Function in Childhood and Adolescence: Development and Plasticity. Child Development Perspectives, 6(4), 354-360. 
